\title{
Concurrent Validity of Digital Image-based Postural Assessment as a Method for Measuring Thoracic Kyphosis: A Cross- Sectional Study of Healthy Adults
}

\author{
Tássia S. Furlanetto, PhD, Cláudia T. Candotti, PhD, Julianna A. Sedrez, PhD, Vinícius H. Dutra, \\ Adriane Vieira, PhD, and Jefferson F. Loss, PhD
}

\begin{abstract}
Objective: To analyze the concurrent validity of the Digital Image-based Postural Assessment (DIPA) method for identifying the magnitude and classification of thoracic kyphosis in adults.

Methodology: On the same day and in the same place, thoracic kyphosis was assessed in 68 adults using 2 methods: the DIPA software protocol and radiography. The DIPA software provided angular values of thoracic kyphosis based on trigonometric relations, while with the radiograph, the curvature was calculated using the Cobb method. The following tests were applied in the statistical analysis: Pearson's correlation, Bland-Altman's graphic representation, root mean square error, and receiver operating characteristic (ROC) curve; $\alpha=0.05$. The reference angular values for the standard thoracic posture used in DIPA were determined with the ROC curve based on the Cobb angles.

Results: The correlation between the angles obtained for thoracic kyphosis using the DIPA and Cobb methods was found to be high $(\mathrm{r}=0.813, P<.001)$, and the accuracy was $\pm 4^{\circ}$. According to Bland-Altman's representation, the magnitudes provided by the DIPA software were in agreement with those of the Cobb method. In reference values for determining the standard posture of the thoracic spine, the ROC curve indicated good accuracy in diagnosing a decrease in thoracic kyphosis (with a value of $33.9^{\circ}$ ) and excellent accuracy in diagnosing thoracic hyperkyphosis (with a value $39.9^{\circ}$ ) when using DIPA.

Conclusion: The DIPA postural assessment method is valid in the sagittal plane for identifying the magnitude of thoracic kyphosis in adults. Furthermore, it is accurate in diagnosing alterations in thoracic kyphosis. (J Manipulative Physiol Ther 2020;43;93-99)
\end{abstract}

Key Indexing Terms: Kyphosis; Validity of Results; Photogrammetry

\section{INTRODUCTION}

According to the American Society for Photogrammetry and Remote Sensing, ${ }^{1}$ photogrammetry is the art, science, and technology of obtaining reliable information about physical objects and the environment through processes of recording, measuring, and interpreting images. Researchers and health care professionals have used photogrammetry to identify postural alteration or to follow up treatment. ${ }^{2}$ When used to assess the spine, it provides angular measures of the sagittal

School of Physical Education, Physiotherapy and Dance, Federal University of the Rio Grande do Sul, Porto Alegre, RS, Brazil.

Corresponding author: Jefferson F. Loss, School of Physical Education, Physiotherapy and Dance, Federal University of the Rio Grande do Sul, Rua Fernando Osorio, 1887, Porto Alegre, RS, Brazil.

(e-mail: jefferson.loss@ufrgs.br).

Paper submitted July 27, 2018; in revised form December 21, 2018; accepted March 29, 2019.

$0161-4754$

(C) 2020 by National University of Health Sciences.

https://doi.org/10.1016/j.jmpt.2019.03.010 spinal curves. ${ }^{3,4}$ The use of photogrammetry undoubtedly contributes to reducing exposure to radiation ${ }^{5}$ and thus enables the monitoring of postural treatment, ${ }^{2}$ such as scoliosis ${ }^{6}$ or cervical posture in mouth-breathing individuals.

A recent systematic review of the noninvasive methods of assessing the sagittal spine showed that studies using photogrammetry presented similar data collection protocols, while differing considerably regarding data analysis processes. ${ }^{8}$ In general, postural assessment protocols use different software and mathematical procedures to measure sagittal curves. ${ }^{3,4,9-11}$ Hence, the authors suggested that each new proposed method be submitted to appropriate validation procedures.

Among the methods that use photogrammetry in combination with computer techniques are the DIPA (Digital Image-based Postural Assessment) software and protocol, which together allow the assessment of different body segments in the sagittal and frontal planes, thus providing clinical information on posture in individuals. ${ }^{12,13}$ Previous studies have confirmed the reproducibility and validity of linear spine measurements in the sagittal and frontal planes, ${ }^{12}$ and angular spine measurements in the frontal plane, ${ }^{13}$ 
obtained using the DIPA software. However, there is a need to validate the angular values and clinical diagnosis obtained with the DIPA software for the spinal curvatures in the sagittal plane.

Therefore, the present study aims to analyze the concurrent validity of the DIPA postural assessment method in relation to radiography for identifying the magnitude and classification of thoracic kyphosis in adults. Based on our previous studies, ${ }^{12-14}$ our hypothesis is that the magnitude of the angle of thoracic kyphosis measured using the DIPA software will agree with that measured using radiography. Similarly, it is thought the DIPA software will provide an accurate classification of the thoracic kyphosis. Hence, this tool can be used on a large scale, for example, to conduct screening for postural alterations in schools, public heath centers, and sports teams, and for research purposes. In the clinical environment, this software will be useful for evaluating and measuring changes due to spinal treatments. For instance, the postural report provided by the DIPA software can assist the clinical management of patients with musculoskeletal disorders, since it provides patients with visual feedback of subtle changes in their posture.

\section{Methods}

\section{Participants}

The participants were recruited from 2 radiography clinics in the metropolitan area in which the university is located. The inclusion criteria were age between 18 and 65 years old and the presentation of a medical prescription for a spinal X-ray. The exclusion criteria were the presence of spina bifida, 6 lumbar vertebrae, diseases or disabilities that affect orthostatism, previous spinal surgery, and unclear radiographic images. All the participants answered a clinical questionnaire designed to identify possible spinal pathologies. This study was approved by the research ethics committee of the university, and the participants signed an informed consent term.

\section{Sample Size Estimation}

The sample size was calculated using $G^{*}$ power software (version 3.1.7), based on the z-test family (Pearson's correlation for dependent samples), assuming 1 tail (the correlation is always expected to be positive), $\mathrm{r}=.4$ as the null hypothesis value (any value less than 0.40 would be considered clinically unacceptable), an expectation of moderate correlation $(\mathrm{r}=.7), \alpha=0.05$, and $80 \%$ of power, resulting in a minimal sample of 60 participants. Allowing for dropouts, 68 individuals were included in the initial sample.

\section{Experimental Design}

Each participant underwent 2 evaluations of the spine, one with the DIPA protocol and another with radiography. For both evaluations, the participants wore appropriate clothing such as a bikini, a top, a bra, shorts, or swimming trunks. In the orthostatic position, the participants were palpated to identify the following anatomical reference points: spinal processes of C7, T1, T2, T4, T6, T8, T10, T12, L2, L4, and S2 (Fig 1A), on which specific reflective markers were placed to facilitate photogrammetry, as required by the DIPA protocol.

After each, the participant was positioned for a single photographic register in the sagittal plane, with the lower limbs together, respecting the postural valgus/varus pattern
A

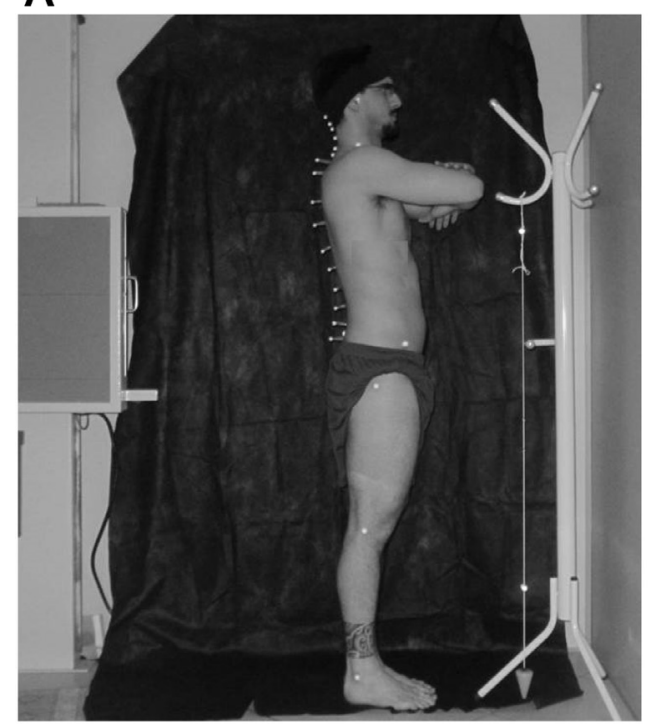

B

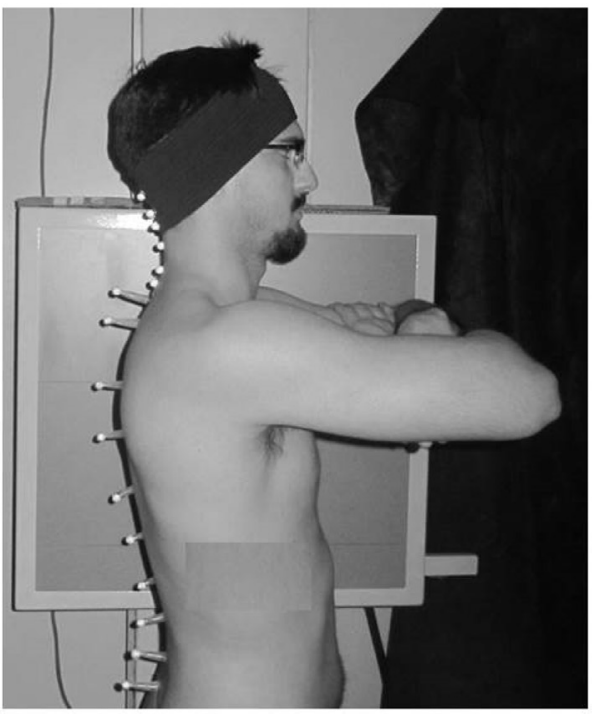

Fig I. The participant in the orthostatic position for the $(A)$ photographic register and $(B)$ radiograph of the thoracic kyphosis. In both situations, the anatomical reference points are identified on the skin surface. 
of each participant, knees extended, shoulders and elbows folded in front of the chest (Fig 1A). For this purpose, the guidelines adopted in previous studies were used. ${ }^{12,13}$ All the evaluation procedures were carried out by the same trained evaluator with more than 10 years' experience in postural assessment using the DIPA protocol in the research environment.

Immediately following the photographic register, in the same room, a radiograph of the thoracic kyphosis was taken (Fig 1B). This was conducted by a trained radiological technician, with the participant in the sagittal plane, maintaining the same position adopted for the photogrammetry.

\section{Outcome Measures}

The photographic images were then transferred to a microcomputer and analyzed using DIPA 3.4 software, which provides an angular value for the thoracic kyphosis, based on a third-degree polynomial interpolated in the region of the points representing C7 to S2 (Fig 2). The angle between the tangents that pass through T2 and T12 provides the measure of thoracic kyphosis (Fig 2A). ${ }^{14}$

The angular value of the thoracic kyphosis in the radiograph was obtained using the 2-line Cobb method calculated by applying an algorithm in MATLAB 7.9. software. ${ }^{15,16}$ To calculate the angle, 2 straight lines were traced, one following the upper edge of the cranial vertebra (T5) and the other following the lower edge of the caudal vertebra (T12), which together represent the limits of the curvature of interest (Fig 2B). The Cobb angle is formed at the point where these 2 lines cross. ${ }^{15,16}$ The data from the DIPA software and the radiograph images were analyzed by the same evaluator, previously trained in both methods. The evaluator was blind regarding the identification of the data.

Based on the Cobb angle value, the thoracic kyphosis in the radiograph was classified according to the standard reference values. ${ }^{17}$ Therefore, each participant in the study sample could be classified into 1 of 3 different groups: (1) normal kyphosis, Cobb angle from $33.7^{\circ}$ to $40.3^{\circ}$; (2) thoracic hyperkyphosis, Cobb angle greater than $40.3^{\circ}$; and (3) decrease in thoracic kyphosis, Cobb angle less than $33.7^{\circ}$.

\section{Statistical Analysis}

The statistical treatment was conducted using SPSS software, version 20, in which the descriptive and inferential analyses of the data were performed. The descriptive analysis is presented as mean and standard deviation (SD) of the angular values obtained from the DIPA software and radiograph. For the inferential analysis, the Kolmogorov-Smirnov test was used to confirm the normality of the data. To identify the concurrent validity of the angular value for the thoracic spine obtained using the DIPA software, the results were compared with the gold standard, that is, the Cobb angles based on radiographs. Thus, the following statistical procedures were performed to compare the angular values obtained using the Cobb and DIPA methods: (1) Pearson's moment-product correlation; (2) Bland-Altman graphic representation, to analyze the agreement; (3) Student's $t$ test, to analyze the differences; and (4) calculation of the root mean square (RMS) error to analyze the accuracy.
A

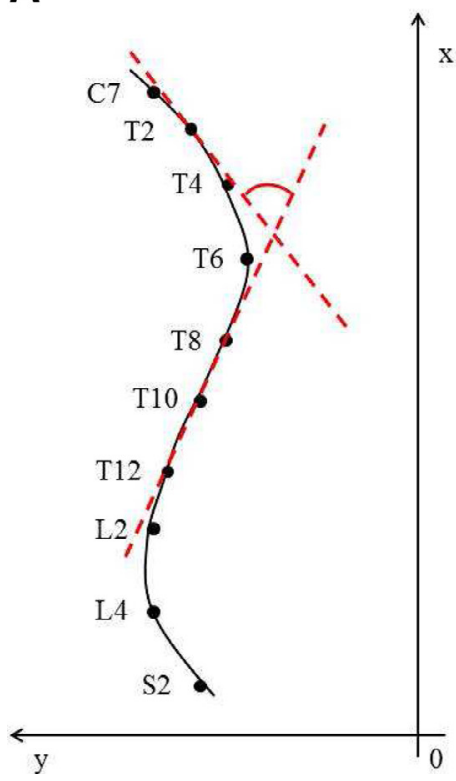

B

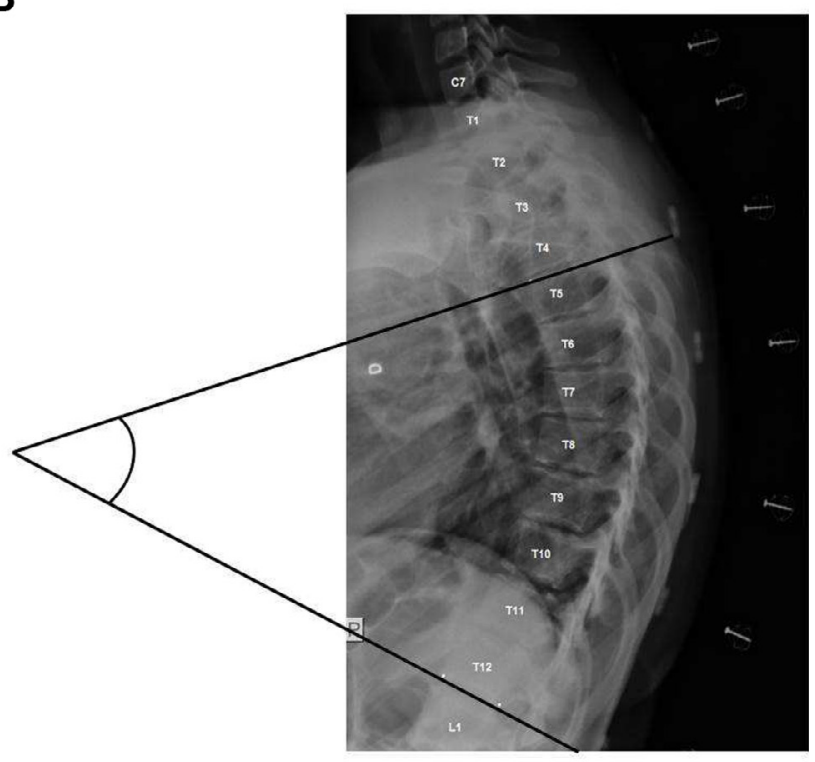

Fig 2. Calculating the angle of thoracic kyphosis (A) provided by the DIPA software and (B) based on radiograph. DIPA, Digital Image-based Postural Assessment. 


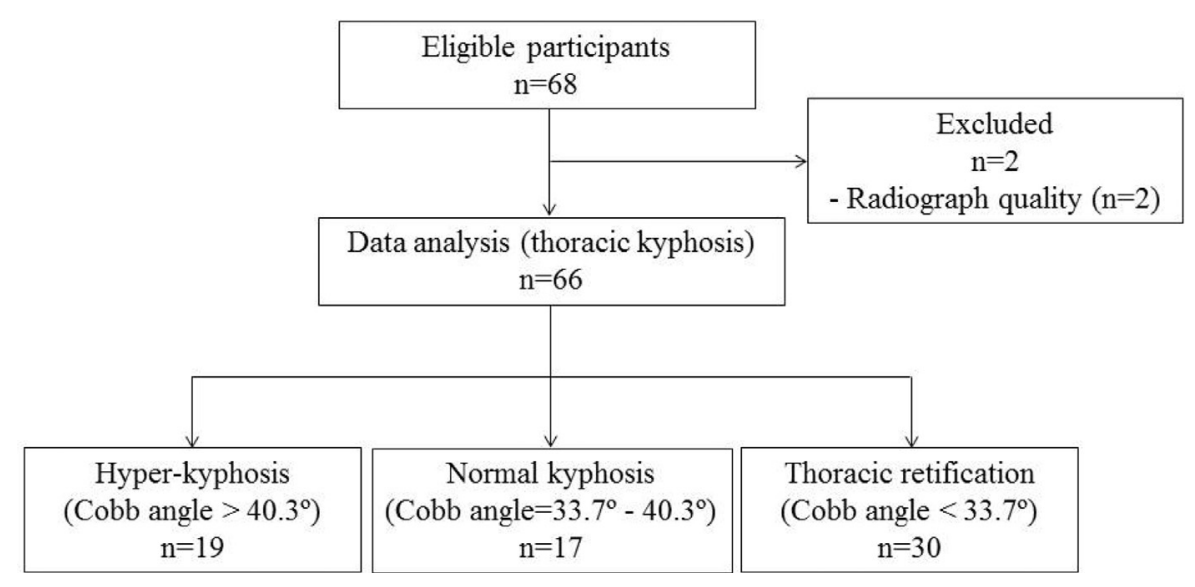

Fig 3. Flow diagram showing the characteristics of the participants.

To identify the concurrent validity of the postural classification provided by the 2 methods (DIPA software and radiograph), the receiver operating characteristic (ROC) curve was used to analyze the accuracy of the diagnosis regarding the changes to the thoracic kyphosis, in which the area under the curve, the cutoff points, and the sensitivity and specificity of the DIPA software were calculated. In this analysis, the accuracy test was divided into (1) capacity to diagnose a decrease in thoracic kyphosis, and (2) capacity to diagnose thoracic hyperkyphosis.

The $\mathrm{r}$ value correlations were classified as very low $(<0.1)$, low (0.1-0.3), moderate (0.3-0.5), high (0.5-0.7), very high $(0.7-0.9)$, and almost perfect $(>0.9) .{ }^{18}$ The area under the ROC curve, which determines the diagnostic accuracy regarding alterations to the thoracic kyphosis, was classified as excellent $(>0.9)$, good $(0.8-0.89)$, regular $(0.7-0.79)$, poor $(0.6-$ $0.69)$, and rejected (0.5-0.59). ${ }^{19}$ A significance level of 0.05 was adopted for all the tests.

\section{RESULTS}

Of the 68 participants who participated in the study, 2 were excluded due to the poor quality of the radiographic images. The average age, body mass, and height were $36.5 \pm$ 15.9 years, $69.7 \pm 14.1 \mathrm{~kg}$, and $1.7 \pm 0.1 \mathrm{~m}$, respectively. Figure 3 is a flow diagram showing the characteristics of the participants.

The angular values provided by the DIPA software are similar to those obtained using the Cobb angles. The correlation between both methods was very high, at $8.2^{\circ} \mathrm{RMS}$ error between angles, and no statistical difference was found between the measurements (Table 1).

The Bland-Altman graphic method shows the agreement between the Cobb and DIPA angles (Fig 4). The mean difference between the methods was $0^{\circ}$ with limits of agreements ( \pm 2 SD) of $16.7^{\circ}$. Only 2 measurements were outside the limits of agreement.

The accuracy of the DIPA software in diagnosing abnormal thoracic kyphosis was assessed using the ROC curve (Fig 5). For the diagnosis of thoracic hyperkyphosis, the area under the ROC curve (Fig 5A) was $0.900(P<.001)$, which indicates excellent accuracy, $78.9 \%$ of sensitivity and $80.9 \%$ specificity, with a cutoff point of $39.9^{\circ}$. For the diagnosis of a decrease in thoracic kyphosis, the area under the ROC curve (Fig 5B) was $0.888(P<.001)$, which indicates good accuracy, $86.1 \%$ of sensitivity and $83.3 \%$ specificity, with a cutoff point of $33.9^{\circ}$.

Using the ROC curve, it was possible to determine the standard postural reference values for the angles provided by the DIPA software when assessing thoracic kyphosis. Table 2 summarizes the normality reference values for both the Cobb and DIPA angles.

\section{DISCUSSION}

According to the findings of the present study, our hypothesis is accepted, since the DIPA software was found to be valid when used to identify the magnitude of the thoracic kyphosis in adults, and accurate when classifying alterations in thoracic kyphosis.

Table I. Mean and SD ( $n=66)$, Pearson's Coefficient Correlation (r), Student $t$ Test $(t)$, and RMS Error of the DIPA and Cobb Angles for Thoracic Kyphosis

\begin{tabular}{llllll}
\hline Measurement Method & Mean $\pm \mathrm{SD}$ & $r$ & Mean Difference $(95 \% \mathrm{CI})$ & $t$ & RMS Error \\
\hline DIPA $\left(^{\circ}\right)$ & $35.7 \pm 11.6$ & $0.813(P<.001)$ & $0.06(-1.98$ to 2.09$)$ & $0.055(P=.956)$ & 8.2 \\
Cobb $\left({ }^{\circ}\right)$ & $35.7 \pm 14.1$ & & & \\
\hline
\end{tabular}




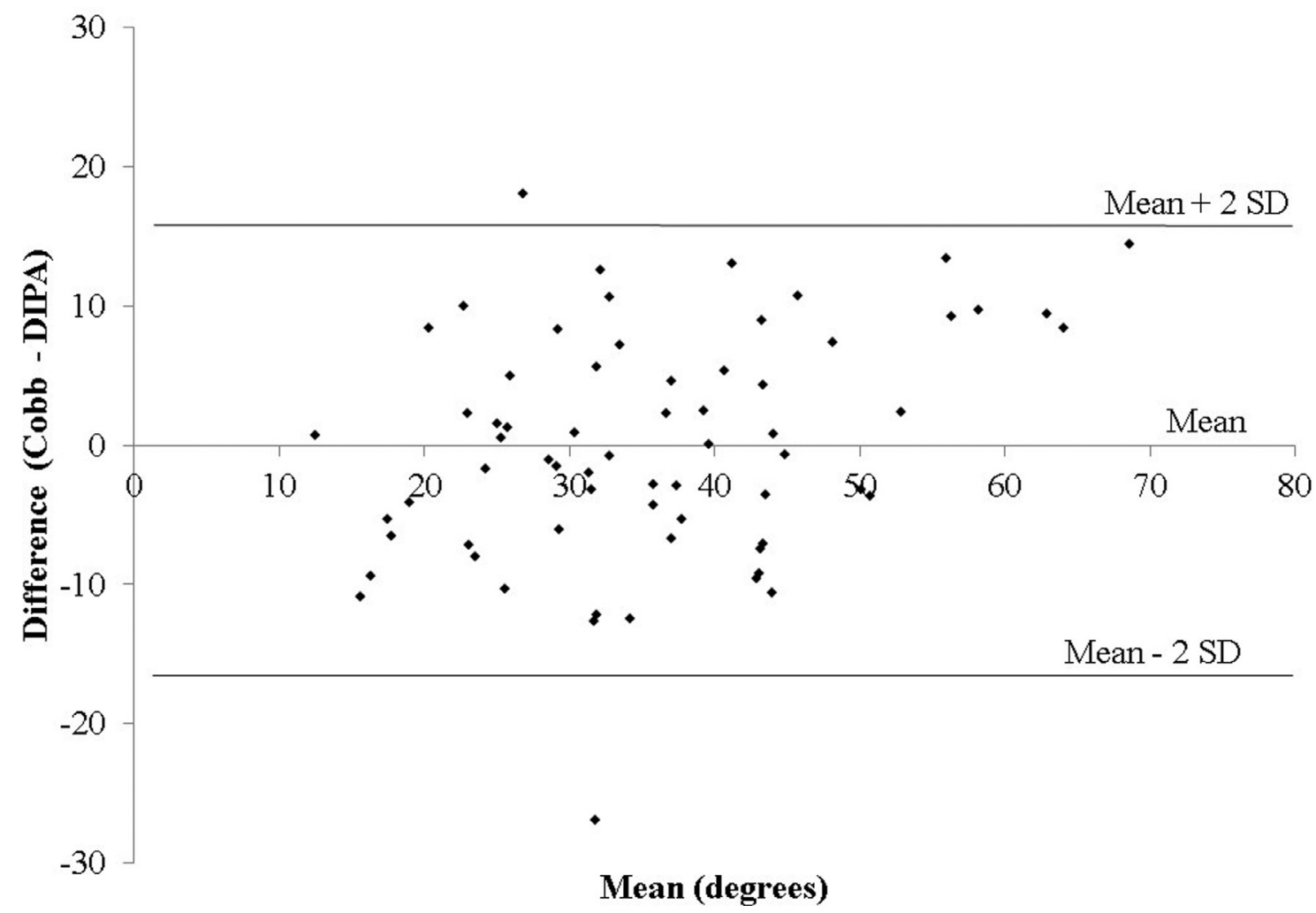

Fig 4. Bland-Altman plot: agreement between the DIPA and Cobb angles for thoracic kyphosis. DIPA, Digital Image-based Postural Assessment.
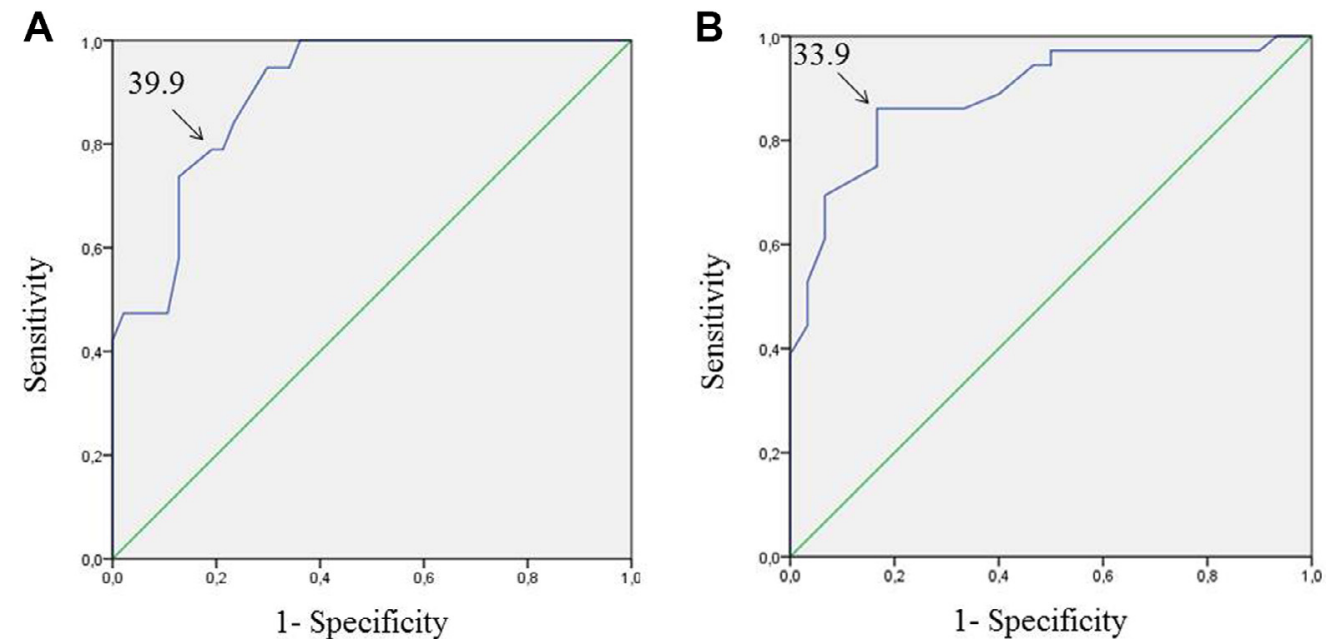

Fig 5. ROC curve for diagnosis of (A) thoracic hyperkyphosis and (B) decrease in thoracic kyphosis using the DIPA angles for thoracic kyphosis. DIPA, Digital Image-based Postural Assessment; ROC, receiver operating characteristic.

The correlation found in relation to the gold standard and the small RMS errors demonstrate the great capacity of the DIPA software in assessing thoracic kyphosis. When using the Cobb method, there may be an error of $\pm 5^{\circ}$ due to the inherent variability of the exam and the evaluator. ${ }^{20}$
Therefore, by comparison, the RMS error of $8^{\circ}$ found in this study demonstrates that the random error between the Cobb and DIPA is satisfactory, given that the variation may be $\pm 4^{\circ}$. Clinically, a difference of up to $5^{\circ}$ is considered merely an exam or evaluator measurement error, while differences of 
Table 2. Normality Reference Values From the DIPA Software and Radiograph for Thoracic Kyphosis

\begin{tabular}{ll}
\hline Method & Normality Range \\
\hline DIPA & $33.9^{\circ}-39.9^{\circ}$ \\
Cobb $^{\text {a }}$ & $33.7^{\circ}-40.3^{\circ}$ \\
\hline
\end{tabular}

DIPA, Digital Image-based Postural Assessment.

${ }^{\text {a }}$ Values from Furlanetto et al. ${ }^{17}$

over $5^{\circ}$ are considered true and important changes in curvature. ${ }^{20}$ Supporting this result, the Bland-Altman plot (Fig 4) demonstrates the random nature of the error between the methods, conforming to the expectation that $95 \%$ of the measurements will be located between the limits of agreement (mean \pm 2 SDs). However, these limits of agreement denote the difference between the results found using the gold standard and the DIPA when measuring thoracic curvature, which could lead to diagnostic error. Based on ROC curve results (Fig 5), this error can be estimated around 20\%, which may be considered acceptable, considering the benefits of this noninvasive method.

Photogrammetry uses the representation of the spinal processes on the skin surface, while the Cobb method uses the vertebral bodies to calculate the magnitude of the spinal curvatures. Anatomically, the spinal processes present an angle of inclination in relation to the vertebral body. ${ }^{21}$ Thus, although photogrammetry has been used to model the outline of the trunk, it can also provide additional information to that obtained using radiographs. ${ }^{9}$ Although the use of different anatomical reference points has been considered a problem, ${ }^{14}$ in the present study the skin surface-based evaluation method was shown to produce similar results to the internal-based method.

Other studies using photogrammetry have also validated the methods adopted by providing accurate results in the evaluation of thoracic kyphosis. ${ }^{3,22}$ However, the values provided by these studies have no clinical significance, since they are not accompanied by standard reference values, preventing postural diagnosis. Thus, an important advantage of using the DIPA software compared with other photogrammetric methods is its diagnostic capacity, since the DIPA software provides a postural classification of the spine. Some software programs, such as PAS/SAPO ${ }^{10}$ and the ALCimagem-2000, ${ }^{11,23,24}$ calculate the angle of the sagittal curvature of the spine based on 2 or 3 points palpated and marked on the spine or other anatomic structures. However, these angles do not provide clinical significance in evaluation. For example, a curvature of $45^{\circ}$ measured using these programs does not necessarily represent thoracic hyperkyphosis, which would be the classification indicated by both the Cobb and DIPA methods. Regarding the classification of the spinal curvature, the diagnostic capacity of the DIPA software in identifying increased or decreased magnitudes of thoracic kyphosis is excellent, providing a suitable range of reference values for normality, very similar to that found using the Cobb angle. ${ }^{17}$

Finally, the literature has shown the DIPA software is reproducible, ${ }^{12}$ and its current version is freely available to users. The results of the present study demonstrate its validity for identifying the magnitude of thoracic kyphosis in adults, as well as providing diagnostic classification of thoracic kyphosis. However, the present study has some limitations, such as the impossibility of carrying out panoramic radiographies of the entire spine, thus impeding the validity of the DIPA software in evaluating cervical and lumbar lordosis. These will be the focus of future studies.

Hence, many health professionals may benefit from adopting this easy-to-use and radiation-free tool, which provides precise, exact, quantitative, and descriptive/classificatory information regarding the thoracic kyphosis of individuals. Thus, some applications of the DIPA software can be listed: physiotherapists and chiropractors can use this method to evaluate patients and follow up treatments in clinical practice; physical education teachers can apply this method to conduct postural screening, so that any alteration diagnosed at school age can be referred for a more detailed evaluation and possible early treatment; physical education teachers can also use this method to prevent sports injuries; and many students and professors in the area of body posture will be able to fruitfully use it in scientific research.

\section{CONCLUSION}

The postural assessment method developed in the DIPA software is valid in the sagittal plane for identifying the magnitude of thoracic kyphosis in adults, as well as providing an accurate diagnostic classification of the thoracic spine posture.

\section{Funding SOURCES AND CONFLICTS OF INTEREST}

No funding sources or conflicts of interest were reported for this study.

\section{CONTRIBUTORSHIP INFORMATION}

Concept development (provided idea for the research): T.S.F., C.T.C., J.F.L.

Design (planned the methods to generate the results): T.S.F., C.T.C., J.F.L., J.A.S., V.H.D.

Supervision (provided oversight, responsible for organization and implementation, writing of the manuscript): T.S.F., C.T.C., J.F.L., A.V.

Data collection/processing (responsible for experiments, patient management, organization, or reporting data): T.S.F., J.A.S., V.H.D. 
Analysis/interpretation (responsible for statistical analysis, evaluation, and presentation of the results): T.S.F., J.A.S., V.H.D.

\section{Practical Applications}

- The DIPA software will can be used in clinical practice, sports and school assessments, and scientific research.

- The DIPA software is easy to use; free; and precise, exact, and quantitative.

- This instrument describes and classifies information regarding the thoracic spine of individuals.

\section{REFERENCES}

1. American Society for Photogrammetry and Remote Sensing. What is ASPRS? Available at: https://www.asprs.org/organi zation/what-is-asprs.html. Accessed September 20, 2018.

2. Furlanetto TS, Sedrez JA, Candotti CT, Loss JF. Photogrammetry as a tool for the postural evaluation of the spine: a systematic review. World J Orthop. 2016;7(2):136-148.

3. Edmondston SJ, Christensen MM, Keller S, Steigen LB, Barclay L. Functional radiographic analysis of thoracic spine extension motion in asymptomatic men. J Manipulative Physiol Ther. 2012;35(3):203-208.

4. Letafatkar A, Amirsasan R, Abdolvahabi Z, Hadadnezhad M. Reliability and validity of the AutoCAD software method in lumbar lordosis measurement. J Chiropr Med. 2011;10:240247.

5. Barrett E, McCreesh K, Lewis J. Reliability and validity of non-radiographic methods of thoracic kyphosis measurement: a systematic review. Man Ther. 2014;19:10-17.

6. Kotwicki T, Negrini S, Grivas TB, et al. Methodology of evaluation of morphology of the spine and the trunk in idiopathic scoliosis and other spinal deformities-6th SOSORT consensus paper. Scoliosis. 2009;4(26).

7. Milanesi JM, Borin G, Corrêa EC, da Silva AM, Bortoluzzi DC, Souza JA. Impact of the mouth breathing occurred during childhood in the adult age: biophotogrammetric postural analysis. Int J Pediatr Otorhinolaryngol. 2011;75(8):999-1004.

8. Sedrez JA, Candotti CT, Furlanetto TS, Loss JF. Non-invasive postural assessment of the spine in the sagittal plane: a systematic review. Motricidade. 2016;12(2):140-154.

9. Saad KR, Colombo AS, Ribeiro AP, João SM. Reliability of photogrammetry in the evaluation of the postural aspects of individuals with structural scoliosis. $J$ Bodyw Mov Ther 2012;16(2):210-216.

10. Ferreira MD, Maldonado EP, Burke TN, Marques AP, Alves EG. Postural assessment software (PAS/SAPO): validation and reliability. Clinics. 2010;65(7):675-681.

11. Iunes DH, Bevilaqua-Grossi D, Oliveira AS, Castro FA, Salgado HS. Comparative analysis between visual and computerized photogrammetry postural assessment. Rev Bras Fisioter. 2009;13(4):308-315.

12. Furlanetto TS, Candotti CT, Sedrez JA, Noll M, Loss JF. Evaluation of the precision and accuracy of the DIPA software postural assessment protocol. Eur J Physiother. 2017;19(4): 179-184.

13. Furlanetto TS, Candotti CT, Comerlato T, Loss JF. Validating a postural evaluation method developed using a digital image-based postural assessment (DIPA) software. Comput Methods Programs Biomed. 2012;108(1):203-212.

14. Furlanetto TS, Oliveira EBC, Candotti CT, et al. Developing and validating prediction equations for the spinal internal curve angles based on the skin surface. J Manipulative Physiol Ther. 2017;40(9):692-699.

15. Goh S, Price RI, Leedman PJ, Singer KP. A comparison of three methods for measuring thoracic kyphosis: implications for clinical studies. J Rheumatol. 2000;39(3):310-315.

16. Mac-Thiong JM, Pinel-Giroux FM, Guise JA, Labelle H. Comparison between constrained and non-constrained Cobb techniques for the assessment of thoracic kyphosis and lumbar lordosis. Eur Spine J. 2007;16(9):1325-1331.

17. Furlanetto TS, Sedrez JA, Candotti CT, Loss JF. Reference values for Cobb angles when evaluating the spine in the sagittal plane: a systematic review with meta-analysis. Motricidade. 2018;14(2-3):115-128.

18. Hopkins WG.A new view of statistics: a scale of magnitudes for effect statistics. Internet Society for Sport Science. Available at: http://www.sportsci.org/resource/stats/. Accessed July 27, 2018.

19. Câmara FP. Psychiatry and statistics V: validation of diagnostic procedures using the R.O.C. curve. Psychiatry On Line Brasil. 2009;14(4). [in Portuguese] .

20. Vrtovec T, Pernus F, Likar B. A review of methods for quantitative evaluation of spinal curvature. Eur Spine J. 2009;18 (5):593-607.

21. Gilad I, Nissan M. Sagittal evaluation of elemental geometrical dimensions of human vertebrae. J Anat. 1985;143:115-120.

22. Van Niekerk S, Louw Q, Vaughan C, Grimmer-Somers K, Schreve K. Photographic measurement of upper-body sitting posture of high school students: a reliability and validity study. BMC Musculoskelet Disord. 2008;9(113):1-11.

23. Iunes DH, Monte-Raso VV, Santos CBA, Castro FA, Salgado HS. Postural influence of high heels among adult women: analysis by computerized photogrammetry. Rev Bras Fisioter. 2008;12(6):454-459.

24. Iunes DH, Cecílio MBB, Dozza MA, Almeida PR. Quantitative photogrammetric analysis of the Klapp method for treating idiopathic scoliosis. Rev Bras Fisioter. 2010;14(2):133-140. 